\title{
Influence of Stabilizing and Encapsulating Polymers on Antioxidant Capacity, Stability, and Kinetic Release of Thyme Essential Oil Nanocapsules
}

\author{
Ricardo M. González-Reza ${ }^{1,2}{ }^{(D)}$, Humberto Hernández-Sánchez ${ }^{1}(\mathbb{D}$, \\ Maria L. Zambrano-Zaragoza ${ }^{2, * \mathbb{D}}$, Gustavo F. Gutiérrez-López ${ }^{1}$, Alicia Del-Real ${ }^{3}$ (D), \\ David Quintanar-Guerrero ${ }^{4}$ (D) and Benjamín Velasco-Bejarano ${ }^{5}$ \\ 1 Departamento de Ingeniería Bioquímica, Instituto Politécnico Nacional, Escuela Nacional de \\ Ciencias Biológicas, UP Adolfo López Mateos, Ciudad de México CP 07738, Mexico; \\ gonzalez.reza@comunidad.unam.mx (R.M.G.-R.); hhernan1955@gmail.com (H.H.-S.); \\ ggutierrezl@ipn.mx (G.F.G.-L.) \\ 2 Laboratorio de Procesos de Transformación y Tecnologías Emergentes de Alimentos, \\ Nacional Autónoma de México, Facultad de Estudios Superiores Cuautitlán, Universidad Cuautitlán Izcalli, \\ Estado de México, Cuautitlán Izcalli CP 54714, Mexico \\ 3 Centro de Física Aplicada y Tecnología Avanzada, Departamento de Ingeniería Molecular de Materiales, \\ Campus Juriquilla, Universidad Nacional Autónoma de México, Querétaro CP 76230, Mexico; \\ adelreal@unam.mx \\ 4 Laboratorio de Posgrado en Tecnología Farmacéutica, FES-Cuautitlán, Universidad Nacional \\ Autónoma de México, Cuautitlán Izcalli, Estado de México, Cuautitlán Izcalli CP 54745, Mexico; \\ quintana@unam.mx \\ 5 Laboratorio L-122 Sección de Química Orgánica, Departamento de Ciencias Químicas, FES-Cuautitlán, \\ Universidad Nacional Autónoma de México, Cuautitlán Izcalli, Estado de México, \\ Cuautitlán Izcalli CP 54745, Mexico; qfbbenjamin.velascob@cuautitlan.unam.mx \\ * Correspondence: luz.zambrano@unam.mx; Tel.: +52-5556231999 (ext. 39406)
}

Received: 9 November 2020; Accepted: 11 December 2020; Published: 17 December 2020

check for updates

\begin{abstract}
The release kinetics, stability, and antioxidant capacity of thyme essential oil polymeric nanocapsules as a function of encapsulating (poly- $\varepsilon$-caprolactone and ethylcellulose) and stabilizing (polyvinyl alcohol and Pluronic ${ }^{\circledR}$ F-127) polymers were established. Samples were evaluated in terms of particle size, zeta potential, release kinetics, calorimetry, infrared spectra, antioxidant capacity, and diffuse reflectance. The particle size obtained was below $500 \mathrm{~nm}$ in all cases, ensuring nanometric size. Zeta potential as a function of the stabilizing polymer. Encapsulation efficiency was higher in the samples that contained ethyl cellulose (around 70\%), associated with its affinity for the molecules contained in the essential oil. Differential scanning calorimetry revealed a strong dependence on the encapsulating polymers as a function of the melting temperatures obtained. Infrared spectra confirmed that the polymeric nanocapsules had the typical bands of the aromatic groups of thyme essential oil. The antioxidant capacity evaluated is a function exclusively of the active content in the nucleolus of the nanocapsules. Nanoencapsulation was not a significant factor. Diffuse reflectance revealed high physical stability of the dispersions related directly to the particle size and zeta potential obtained (either by ionic or steric effect). These findings confirm favorable characteristics that allow proposing these systems for potential applications in food processing and preservation.
\end{abstract}

Keywords: food nanotechnology; polymeric nanoparticles; natural actives; polyvinyl alcohol;

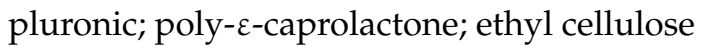




\section{Introduction}

Essential oils are of great commercial interest for their potential applications as fragrances in foods, cosmetics and use as active ingredients in insecticides and the pharmaceutical industry. Their use is sometimes challenging due to low water solubility, volatility, and stability. Micro- and nanoencapsulation of essential oils or their active ingredients are significant for applying essential oils in food and other industries. Thyme essential oil of Thymus vulgaris L. contains molecules of phenolic compounds, such as thymol and carvacrol, which give it its characteristic antioxidant and antimicrobial properties $[1,2]$. The antioxidant capacity of various systems has been studied, as well as the factors that directly influence its numerical value; among these is the composition (if it is an individual component or if it is mixed with other ingredients, whether antioxidants or not), the temperature at which the substance is found if it is in the presence of an atmosphere rich in oxygen or it is in a modified atmosphere, and, finally, the concentration of the antioxidant substance in the medium [3]. The potential application of this essential oil in food has been limited due to its low solubility in water and high volatility. Nanotechnology has allowed nanostructures of bioactive substances like essential oils to increase their solubility and allow targeted delivery, depending on their intended purpose [4]. One way in which these oils can be encapsulated is by forming polymeric nanocapsules that consist of an internal liquid core surrounded by a polymeric membrane [5]. Nanoparticles of this kind can be either lipophilic or hydrophobic, depending on the materials incorporated and the method used to obtain them [6]. For the formation of polymeric nanocapsules by the emulsification-diffusion process, the saturated organic phase, the saturated aqueous phase, and finally, the diluted phase is required [7]. According to the above, the organic phase saturated with water contains the bioactive compound (generally dissolved in an oily core) and the encapsulating polymer. The aqueous phase saturated with the organic phase contains the stabilizing polymer; finally, the dilution is carried out with the unsaturated aqueous phase, usually water. [8]. Polymers commonly used in active substances' encapsulation include poly- $\varepsilon$-caprolactone (PCL) and ethyl cellulose (ETC). PCL is a biodegradable polyester approved by the U.S. Food and Drug Administration (FDA) that significantly impacts lipophilic active substances' encapsulation due to its biocompatibility, slow degradation rate ease of processing [9]. Ethylcellulose is a biocompatible polymer derived from cellulose in which ethyl ether groups replace some of the hydroxyl groups. It is soluble in organic solvents but not in aqueous solutions, so it is highly-recommended for encapsulating lipophilic substances [10]. Polyvinyl alcohol (PVA) stands out among stabilizing polymers as a semi-crystalline polymer that is highly hydrophilic, biodegradable, biocompatible, and has good thermal properties, high water solubility, and good gas permeability, among other properties. PVA is produced by hydrolysis from different degrees of polyvinyl acetate. Its final polymer properties depend on the degree of hydrolysis [11]. Pluronic ${ }^{\circledR} \mathrm{F}-127$ (poloxamer 407) is a nonionic hydrophilic surfactant, a three-block copolymer consisting of a central hydrophobic block of polypropylene glycol flanked by two hydrophilic blocks of polyethylene glycol. Poloxamer is widely used as a stabilizer in nanostructured systems [12]. The choice of polymers for forming nanostructured systems depends directly on the application for which they are proposed. Maybe a feature most important of these systems is the controlled release of active substances. The modeling of the release profiles provides valuable information that can be used to predict storage times, food shelf life, and the formulation of intelligent and active packaging systems [13]. Thyme essential oil nanoencapsulation has been reported with other biopolymers such as chitosan, Arabic gum, casein, maltodextrin, and starch. However, the present proposal for a nanostructured system offers potential application in different branches of food processing, aiming to use biodegradable polymers widely used in the pharmaceutical industry, which is also safe (approved by the FDA) offers controlled release characteristics. These physically stable systems have already been tested in food applications such as edible coatings in fresh-cut fruits, using active substances such as $\beta$-carotene and $\alpha$-tocopherol $[14,15]$. This investigation aimed to establish the physical characteristics, antioxidant capacity, and release profiles obtained for nanostructured thyme essential oil using different encapsulating and stabilizing polymers for potential applications in the food industry. 


\section{Materials and Methods}

\subsection{Materials}

Thyme essential oil $\left(\rho=0.917 \mathrm{~g} / \mathrm{cm}\right.$ at $\left.25^{\circ} \mathrm{C}\right)$ was used to form the oil nucleus. Poly- $\varepsilon$-caprolactone (PCL) $\left(\mathrm{MW} \approx 80 \mathrm{kDa}, \rho=1.147 \mathrm{~g} / \mathrm{cm}^{3}\right.$ at $\left.25^{\circ} \mathrm{C}\right)$ and ethylcellulose (ETC) $(\mu=100 \mathrm{cP}$ in 80:20 toluene/ethanol solution ( $50 \mathrm{~g} / \mathrm{L})$ at $25^{\circ} \mathrm{C}, 48 \%$ ethoxy) were used as encapsulating polymers. Pluronic ${ }^{\circledR}$ F-127 (Poloxamer 407) and polyvinyl alcohol (PVA) (M.W. $\approx 89-98 \mathrm{kDa}, \mu=11.6-15.4 \mathrm{cP}$ in $40 \mathrm{~g} / \mathrm{L} \mathrm{H} \mathrm{H}_{2} \mathrm{O}$ at $\left.20{ }^{\circ} \mathrm{C}\right)$ were the stabilizing agents. ABTS (2,2'-azino-bis-(3-ethylbenzthiazoline)-6-sulfonic acid), DPPH (2,2-diphenyl-1-picrylhydracil) and FRAP (2,4,6-tri (2-pyridyl)-s-triazine) were used for antioxidant capacity measurement. The reagents were purchased from Sigma-Aldrich ${ }^{\circledR}$ (St. Louis, MO, USA). High Performance Liquid Chromatography (HPLC)-grade organic solvents (ethyl acetate for the formation of the nanocapsules and cyclohexane for the release kinetics) used in the present experimental study were purchased by Fermont ${ }^{\circledR}$, Monterrey, Mexico. The rest of reagents were analytical grade.

\subsection{Nanocapsule Preparation}

The emulsification-diffusion method was used to form the nanocapsules that contained thyme essential oil $[7,8]$. Ethyl acetate saturated with water was used as the organic phase, in which the encapsulating polymer (ethylcellulose or PCL) and thyme essential oil were dissolved. The aqueous phase consisted of water saturated with ethyl acetate, which contained the stabilizing polymer (polyvinyl alcohol or Pluronic ${ }^{\circledR}$ F-127). The organic phase and the aqueous phase were subjected to ultra-high agitation in an ultraturrax (T18, IKA ${ }^{\circledR}$ Werke, Staufen, Germany) at $420 \mathrm{~s}^{-1}$ for $10 \mathrm{~min}$. Subsequently, the diffusion was promoted by adding excess water, following the ultra-high agitation to the aforementioned conditions. The excess organic solvent was removed on a rotary evaporator (RV10, IKA ${ }^{\circledR}$ Wilmington, NC, USA) at $9 \mathrm{kPa}$ and $45^{\circ} \mathrm{C}$. Once the samples were obtained, they were stored at $25^{\circ} \mathrm{C}$ for later analysis. The nanocapsules obtained contained $1 \mathrm{~g} / \mathrm{L}$ of thyme essential oil, $0.4 \mathrm{~g} / \mathrm{L}$ of encapsulating polymer, and $4.8 \mathrm{~g} / \mathrm{L}$ of stabilizing polymer.

\subsection{Dynamic Light Scattering and Electrophoretic Movement}

For the analysis of particle size (P.S.) and polydispersity index (PDI), the nanocapsules were diluted in distilled water (1:20) to obtain volume frequency histograms. For the evaluation of the dynamic light scattering, a fixed measurement angle of $273^{\circ}$ was used. The study of the electrophoretic movement in the nanocapsules was expressed as zeta potential $(\zeta)$. For the evaluation of the $\zeta$, the sample was diluted with distilled water of a similar form that the users in the dynamic light scattering measurement was carried out. The aforementioned parameters were analyzed on a Z-sizer 4 (Malvern Ltd., Grovewood Road, UK). Measurements of each parameter were carried out in independent experiments at $25^{\circ} \mathrm{C}$ in triplicate [16].

\subsection{Nanocapsule Morphology}

The nanocapsules' morphology containing thyme essential oil was analyzed using the methodology proposed by González-Reza et al. (2018) [4] by Scanning Electron Microscope (SEM). The images were observed in a scanning electron microscope (Hitachi, SU-8230, Tokyo, Japan) with a BSE + BSE (U) detector.

\subsection{Encapsulation Efficiency (E.E.)}

E.E. was determined by spectrophotometry using a Biospectrophotometer (Eppendorf AG, 22331, Hamburg, Germany) at $275 \mathrm{~nm}$. To physically separate unencapsulated amounts of thyme essential oil 
was employment centrifugation in an ultracentrifuge (Hermle Z323K, Labortechnik GMBH, Wehingen, Germany) for $20 \mathrm{~min}$ at $4{ }^{\circ} \mathrm{C}$ and $18,000 \times g$. E.E. was determined by the following expression:

$$
E E(\%)=\left(\frac{\text { Essential oil concentration retained }}{\text { Total concentration of essential oil }}\right) \times 100
$$

\subsection{Release Kinetics}

Release of thyme oil was carried out according to the methodology proposed by Soares et al. (2016) [17] with some modifications. Briefly, the release profiles of the N.C.s were determined using cyclohexane. The nanoparticles were lyophilized in a vacuum dryer. The resulting powder was resuspended in the cyclohexane according to treatment at a concentration of $1 \mathrm{~g} / \mathrm{L}$ and separated by ultracentrifugation at $18,000 \times \mathrm{g}$ for $20 \mathrm{~min}$ at $5{ }^{\circ} \mathrm{C}$ to remove suspended particles and avoid errors in the measurements. The volume lost periodically by each measurement was replaced. All samples' absorbance was measured and correlated with thyme essential oil calibration curves $(\lambda=275 \mathrm{~nm})$. The concentrations and the percentage of the active ingredient released over time were calculated in a Biospectrophotometer (Eppendorf AG, 22331, Hamburg, Germany). Controls were made by centrifuging $1 \mathrm{~mL}$ of free polymer in the release medium. All nanocapsule release experiments were performed in triplicate for $48 \mathrm{~h}$ at $25^{\circ} \mathrm{C}$.

\subsection{Infrared Spectroscopy}

The infrared absorption spectra of the individual compounds and the nanoparticles obtained were examined by spectroscopy in an I.R. spectrum (PerkinElmer Spectrum 400 IR, Waltham, MA, USA). Samples with a minimum moisture content were placed in the prism for further analysis. Spectra were obtained with an interval of $500-4000 \mathrm{~cm}^{-1}$ and a resolution of $1 \mathrm{~cm}^{-1}$ at $25^{\circ} \mathrm{C}$. The ambient spectrum was taken as a target and reference. The results obtained were analyzed in the equipment's software

\subsection{Thermal Behavior}

The heat flow in the formed nanocapsules, as well as in their components, was obtained in a differential scanning calorimeter (T.A. Instruments, DSC Discovery, New Castle, DE, USA). The working conditions used were a measurement range of -20 to $150^{\circ} \mathrm{C}$ with a heating ramp of $10^{\circ} \mathrm{C} / \mathrm{min}$. The results obtained were analyzed in the equipment's software.

\subsection{Diffuse Reflectance}

The stability of the nanocapsules was determined with a diffuse reflectance using Turbiscan MA2000 equipment (Formulaction, Toulouse, France), according to the measurement protocol proposed by González-Reza et al. (2018) [4] every $8 \mathrm{~min}$ for $24 \mathrm{~h}$, utilizing two synchronous detectors: one for transmission and one for backscatter. Samples were analyzed at $25^{\circ} \mathrm{C}$ [4].

\subsection{Antioxidant Capacity}

The antioxidant capacity of the nanocapsules was determined spectrophotometrically in a BioSpectrometer ${ }^{\circledR}$ (Eppendorf AG, 22331, Hamburg, Germany) using positive control ascorbic acid by ABTS, DPPH, and FRAP. The results were contrasted with a reference curve constructed by plotting absorbance values against 100-1000 $\mu \mathrm{mol}$ concentrations of ascorbic acid. Results were expressed as $\mu \mathrm{mol}$ equivalents of ascorbic acid/g of nanostructured essential oil. Measurements were made in triplicate. 


\subsubsection{ABTS}

The antioxidant capacity determined by ABTS was evaluated according to the measurement protocol reported by Re et al. (1999) [18]. Measurements were made at $25^{\circ} \mathrm{C}$ after 6 min by spectrophotometry at $734 \mathrm{~nm}$.

\subsubsection{DPPH}

The antioxidant capacity determined by DPPH was evaluated according to the measurement protocol reported by Brand-Williams et al. (1995) [19]. Measurements were made at $25^{\circ} \mathrm{C}$ after a $30 \mathrm{~min}$ incubation time in the dark by spectrophotometry at $517 \mathrm{~nm}$.

\subsubsection{FRAP}

The antioxidant capacity determined by FRAP was evaluated according to the measurement protocol reported by Benzie \& Strain (1996) [20]. Measurements were made at $37^{\circ} \mathrm{C}$ after a $30 \mathrm{~min}$ incubation time in the dark by spectrophotometry at $595 \mathrm{~nm}$. A control without a sample was also prepared.

\subsection{Statistical Analyses}

Statistically significant differences were established by ANOVA $(\alpha=0.05)$. All statistical analyses were performed using the Minitab statistical program (Minitab ${ }^{\circledR}$ Statistical Software 19 Inc., Center, PA, USA).

\section{Results}

\subsection{Dynamic Light Scattering, Electrophoretic Movement, and Charging Efficiency}

Table 1 shows the physical parameters obtained for the N.C.s under the different experimental conditions. All the samples were determined to have average particle sizes of $500 \mathrm{~nm}$ within nanometric size.

Table 1. Characterization by laser light scattering, electrophoretic movement, and encapsulation efficiency of polymeric nanocapsules.

\begin{tabular}{ccccc}
\hline Sample & P.S. $(\mathbf{n m})$ & PDI (-) & $\zeta(\mathbf{m V})$ & E.E. (\%) \\
\hline ETC-PVA & $346.97 \pm 0.85$ & $0.09 \pm 0.01$ & $-7.16 \pm 0.28$ & $71.28 \pm 0.68$ \\
ETC-Pluronic $^{\circledR}$ & $266.13 \pm 3.53$ & $0.11 \pm 0.03$ & $-19.77 \pm 0.76$ & $69.79 \pm 2.92$ \\
PCL-PVA & $489.20 \pm 3.93$ & $0.12 \pm 0.01$ & $-5.89 \pm 0.36$ & $60.87 \pm 0.84$ \\
PCL-Pluronic $^{\circledR}$ & $241.37 \pm 2.06$ & $0.12 \pm 0.05$ & $-16.97 \pm 0.83$ & $67.43 \pm 0.91$ \\
\hline
\end{tabular}

The particle size distribution obtained for the different nanostructured systems is shown in Figure 1. The samples presented significant differences $(p \leq 0.05)$ in the encapsulating polymer and stabilizing polymer used. Reviews about P.S. (less than $500 \mathrm{~nm}$ ) of polymeric nanocapsules formed by the emulsification-diffusion method have been reported [6]. In addition to analyzing the nanoencapsulation of bioactive substances in polymers such as poly- $\varepsilon$-caprolactone and ethyl cellulose [4,21]. The polydispersity index values obtained—below 0.15 in all cases $(p>0.05)$ - suggest that the dispersions had a narrow distribution [22].

The $\zeta$ obtained for the samples stabilized with Pluronic ${ }^{\circledR}$ F-127 was higher than that of the samples containing PVA $(p \leq 0.05)$, since in colloidal systems the charge arises from the ionization of the surface groups, adsorption of the material from the active surface, the continuous charges associated with the crystalline structures, or the combination of these mechanisms [23]. The lowest zeta potential values were found in the samples stabilized with PVA since that stabilization occurred as a result of steric effects rather than repulsion effects caused by surface charges [24]. In the case of 
encapsulation efficiency, values ranged from $60-70 \%$ for all samples, highlighting that the highest retention occurred using ethyl cellulose $(p \leq 0.05)$. The results were very near those reported by Galindo-Pérez et al. (2018) [21] to encapsulate essential oils using the emulsification-diffusion method.

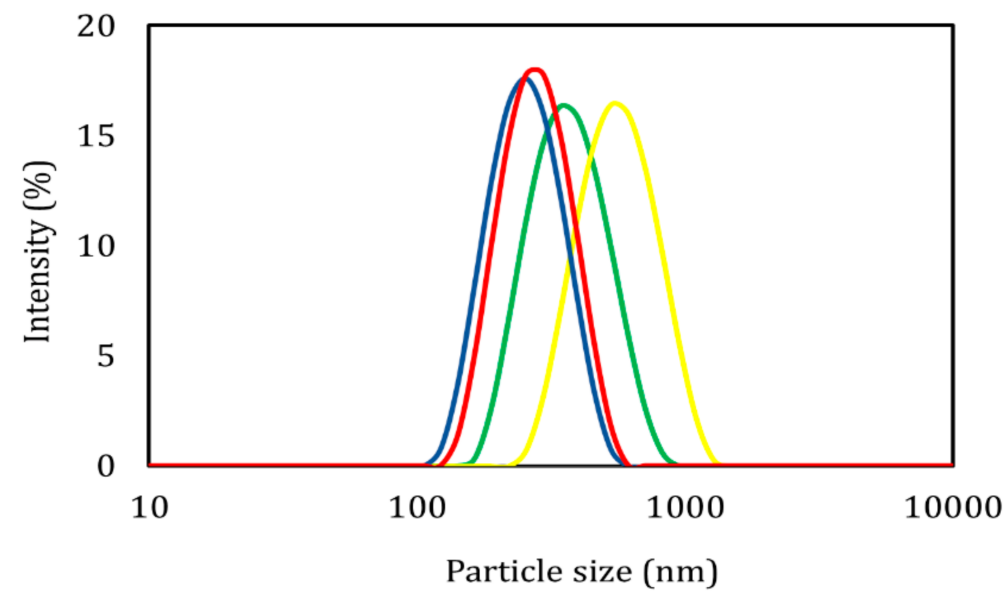

Figure 1. Distribution of the particle size obtained for the different treatments.

\subsection{Nanocapsule Morphology}

Figure 2 shows micrographs that correspond to the polymeric nanocapsules, revealing a distorted, spherical shape with some agglomerations. The capsular structure consists of the polymeric membrane as a layer that surrounds an oily nucleus, as reported by Noronha et al. (2013) [25] for the formation of $\alpha$-tocopherol/PCL nanocapsules by nanoprecipitation.

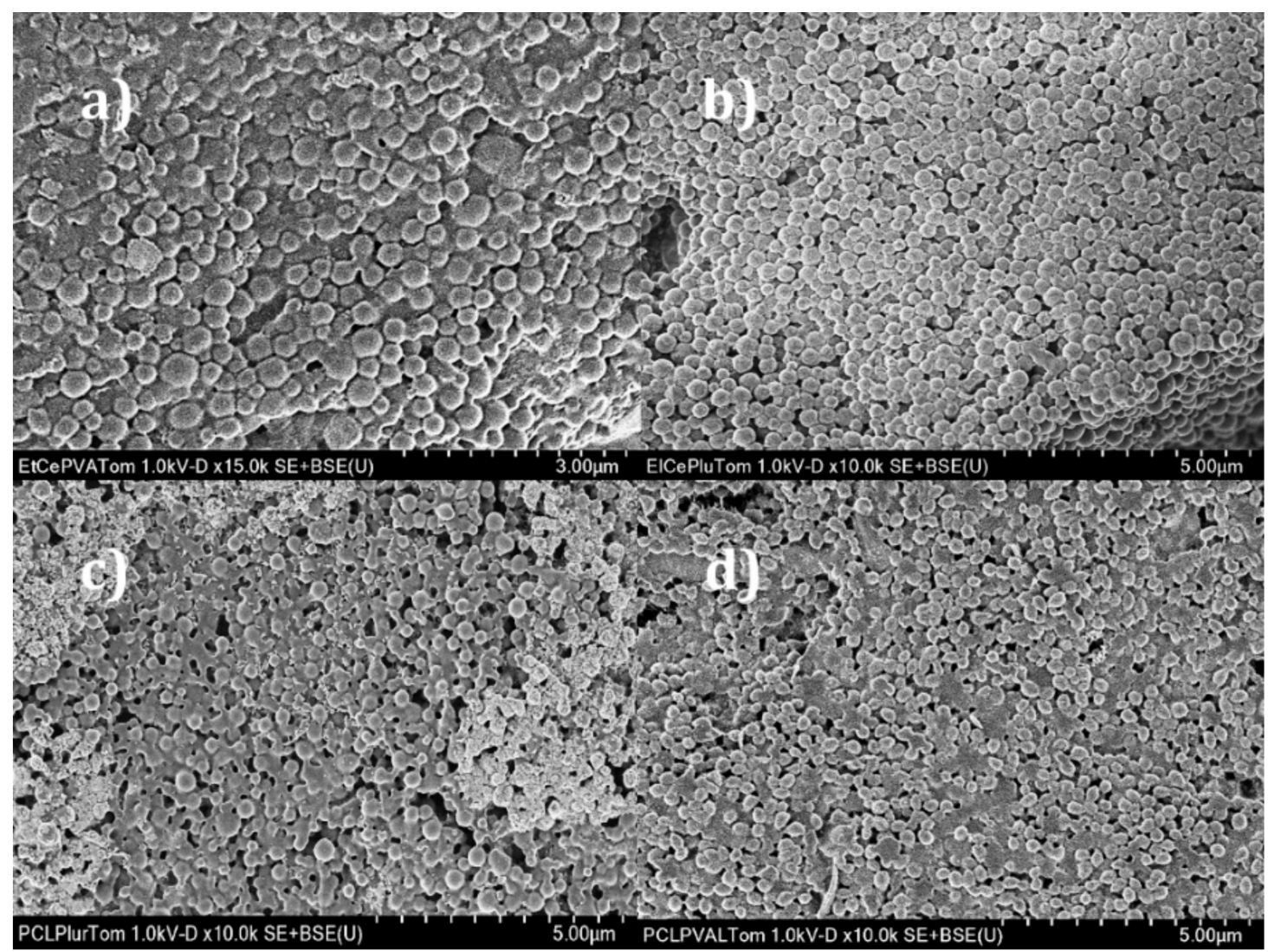

Figure 2. Morphological characterization by SEM of the polymeric nanocapsules: (a) ethyl cellulose-PVA; (b) ethyl cellulose-Pluronic ${ }^{\circledR}$ F-127; (c) PCL-Pluronic ${ }^{\circledR}$ F-127; (d) PCL-PVA. 
The average size of the nanostructure observed by SEM correlated successfully with laser light-scattering's average size. The micrographs obtained reveal regular spherical nanocapsules, and their size agrees with that obtained by dynamic light scattering.

\subsection{Release Kinetics of Nanocapsules}

A wide variety of mathematical models are available to fit bioactive agent release data, most of them presented as nonlinear equations. Figure 3 shows the release profiles of the nanocapsules. The ANOVA revealed that the use of different encapsulating polymers gave a statistically significant effect $(p \leq 0.05)$ on the release of the thyme essential oil, in contrast to the stabilizing polymers $(p>0.05)$.

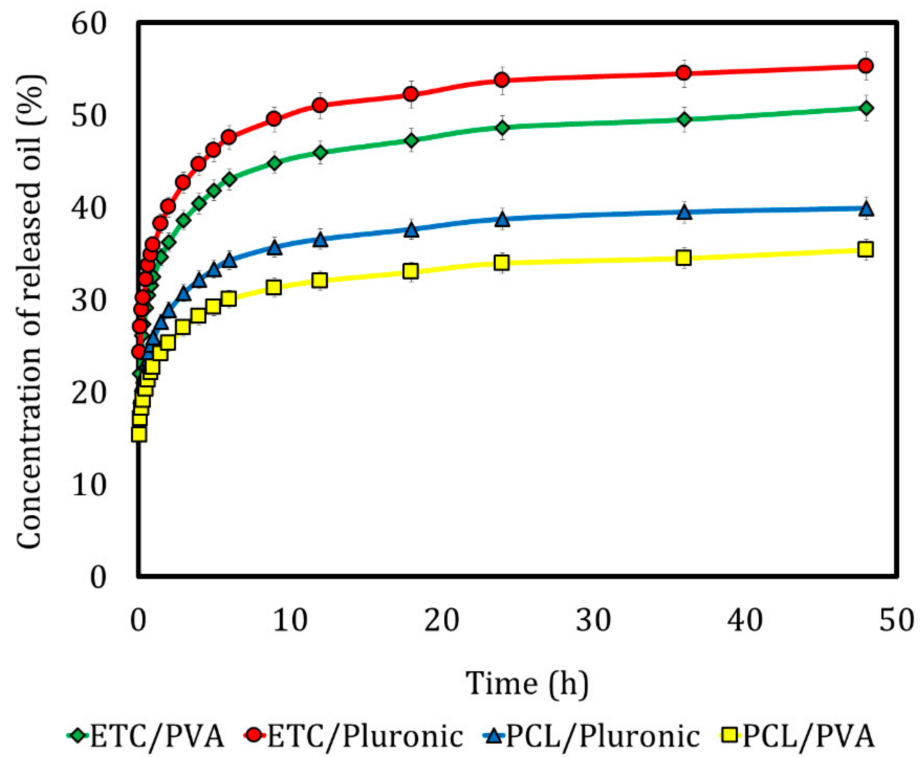

Figure 3. In vitro release profiles obtained for the different polymeric nanocapsules.

Regarding the modeling of the release profiles, five models widely used in the literature were tested to understand the phenomena that predominated in releasing the thyme essential oil in the nanocapsules formed by the diffusion emulsification method. Table 2 shows the kinetic parameters obtained from the thyme essential oil release curves.

Table 2. Models applied to the release profiles in polymeric nanocapsules.

\begin{tabular}{ccccccc}
\hline Sample & Zero-Order & First-Order & Higuchi & Hixon-Crowel & \multicolumn{2}{c}{ Korsmeyer-Peppas } \\
\hline & $\mathbf{R}^{\mathbf{2}}$ & $\mathbf{R}^{\mathbf{2}}$ & $\mathbf{R}^{\mathbf{2}}$ & $\mathbf{R}^{\mathbf{2}}$ & $\mathbf{R}^{\mathbf{2}}$ & $\mathbf{n}$ \\
\hline ETC-PVA & 0.5938 & 0.5116 & 0.8259 & 0.5395 & 0.9830 & 0.1363 \\
ETC-Pluronic $^{\circledR}$ & 0.5797 & 0.5015 & 0.8259 & 0.5281 & 0.9808 & 0.1357 \\
PCL-PVA & 0.5925 & 0.5117 & 0.8259 & 0.5385 & 0.9830 & 0.1360 \\
PCL-Pluronic $^{\circledR}$ & 0.5840 & 0.5049 & 0.8259 & 0.5316 & 0.9815 & 0.1357 \\
\hline
\end{tabular}

The release kinetics modeled by Korsmeyer-Peppas presented a good correlation with all the models studied, as no statistical differences were found among the release constants $(p>0.05)$. Thyme essential oil release studies verified this behavior for chitosan nanocapsules prepared by nanoprecipitation [26]. The percentage of bioactive release can be determined using constant values. The " $\mathrm{n}$ " values in the Korsmeyer-Peppas model provides information on the release mechanism. If $n<0.43$, there is Fickian diffusion [17]. Release studies conducted for polymeric nanocapsules (PCL/ $\beta$-carotene) obtained by the emulsification-diffusion method and applied as an edible coating on fresh-cut melon found that the Korsmeyer-Peppas model indicated a release pattern that can be 
explained by time-dependent Fickian broadcast Zambrano-Zaragoza et al. (2017) [14]. This is consistent with the findings obtained in the present study.

\subsection{Infrared Spectra of the Nanocapsules}

Figure 4a shows the infrared (I.R.) characterization of the thyme essential oil. The typical thyme essential oil absorption peaks detected at 2926 and $2969 \mathrm{~cm}^{-1}$ were caused by the $\mathrm{C}-\mathrm{H}$ stretching vibration of aliphatic methylene for the oil core. The peaks at 1586 and $1466 \mathrm{~cm}^{-1}$ were attributed to the $C=C$ skeletal vibration of the oil's benzene ring. The peaks detected at 1376 and $1108 \mathrm{~cm}^{-1}$ were assigned to the $\mathrm{C}-\mathrm{O}-\mathrm{H}$ flex mode and the -C-O- stretching vibration, respectively. The absorption peak at $947 \mathrm{~cm}^{-1}$ was attributed to the $\mathrm{C}=\mathrm{C}$ stretching vibration or the bending vibration of some hydrogen-containing groups [27].

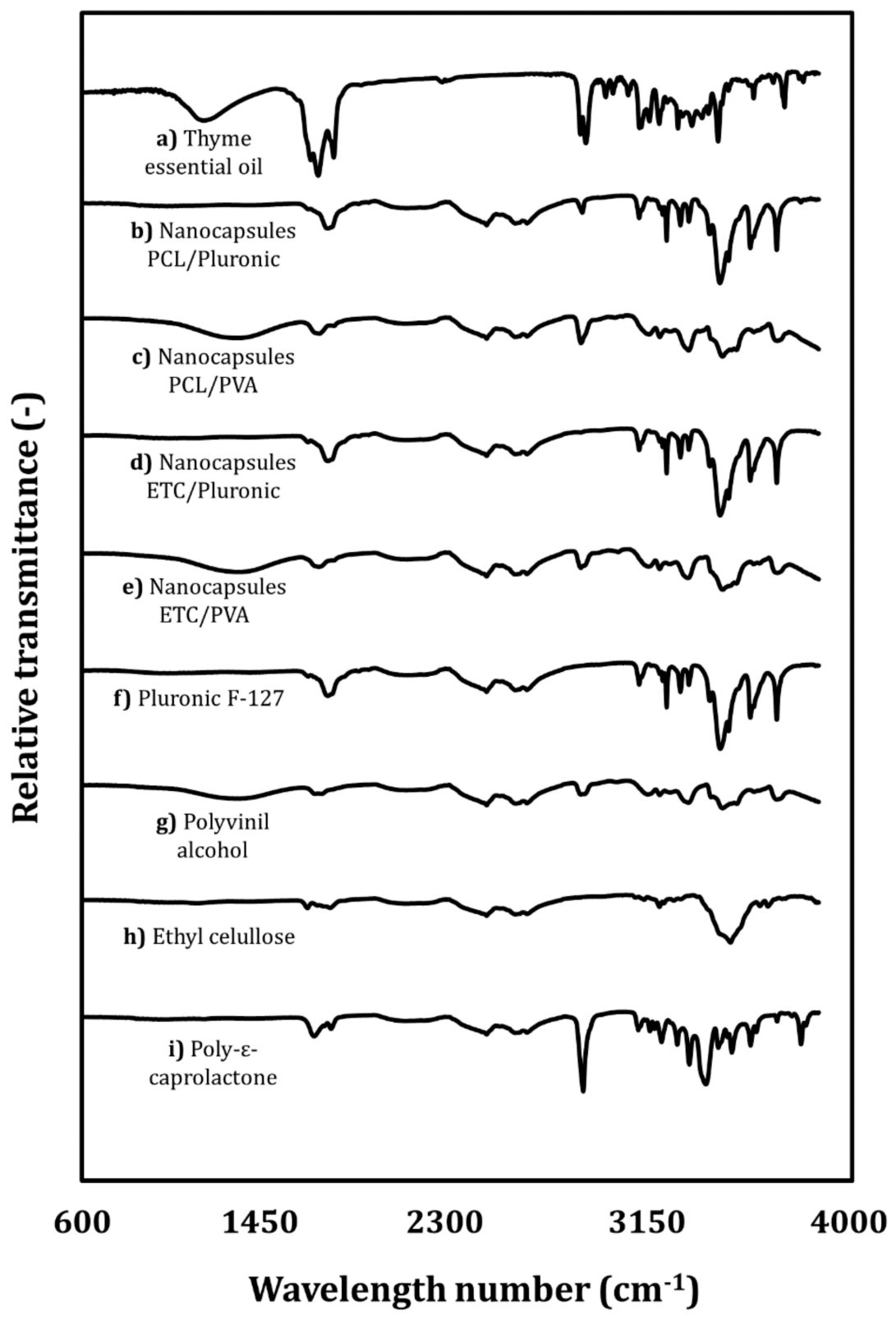

Figure 4. Infrared spectra for (a) thyme essential oil; (b) PCL-Pluronic ${ }^{\circledR}$ F-127; (c) ETC-Pluronic ${ }^{\circledR}$ F-127; (d) PCL-PVA) polymeric nanocapsules; the (e) ETC-PVA)and (f) Pluronic ${ }^{\circledR}$ F-127 stabilizing polymers;

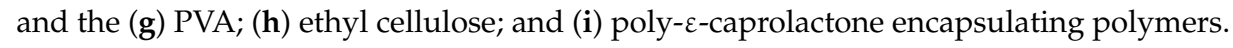


Figure $4 \mathrm{i}$ shows the spectrum of the PCL. Strong bands were as the carbonyl stretch mode around $1721 \mathrm{~cm}^{-1}$. The $1278 \mathrm{~cm}^{-1}$ band is assigned to the C-C and C-O spinal stretch modes in crystalline PCL [28]. The ETC (Figure 4h) presents a weak stretch band at $3480 \mathrm{~cm}^{-1}$ that corresponds to the O.H. bond vibration mode and medium intensity stretch bands at 2974 and $2871 \mathrm{~cm}^{-1}$ that, in turn, correspond to the C-bond vibration modes. C.H. is present in the alkane groups of the ethylcellulose. Typical stretch bands of 1444,1375 , and $1310 \mathrm{~cm}^{-1}$ were found that correspond to the vibratory mode of flexion of the $-\mathrm{CH}_{3}$ groups and corresponding $1444 \mathrm{~cm}^{-1}$ of the $-\mathrm{CH}_{2}$ groups, in addition to the characteristic band at $1052 \mathrm{~cm}^{-1}$ that corresponds to the vibration mode of the stretching of C.O. bonds [10]. The main peaks for Pluronic ${ }^{\circledR}$ F-127 (Figure 4f) were present in the $2883 \mathrm{~cm}^{-1}$ (C.H. stretch), $1342 \mathrm{~cm}^{-1}$ (O.H. stretch), and $1099 \mathrm{~cm}^{-1}$ zones (C.O. stretch) and harmonized with the standard spectra of the aforementioned polymer. In the PVA case (Figure 4g), the bands at 3290 and $2911 \mathrm{~cm}^{-1}$ were attributed to O.H. and $\mathrm{CH}_{2}$ stretching, respectively. Other characteristic bands for PVA appeared at 1428, 1325, 1087, and $944 \mathrm{~cm}^{-1}$ and were attributed to $\mathrm{O}-\mathrm{H}, \mathrm{C}-\mathrm{H}$ flexion, C-O-H flexion, and $\mathrm{C}-\mathrm{O}$ and $\mathrm{CH}_{2}$ balancing, respectively.

The infrared spectra for polymeric nanocapsules were also analyzed. The spectra obtained at the typical absorption peaks of thyme essential oil were detected at 2926 and $2969 \mathrm{~cm}^{-1}$ in all samples. The peaks at 1563 and $1466 \mathrm{~cm}^{-1}$ were attributed to the $C=C$ skeletal vibration of the benzene ring in the oil loaded in the nanocapsules. The peaks at 1374 and $1110 \mathrm{~cm}^{-1}$ were assigned to the $\mathrm{C}-\mathrm{O}-\mathrm{H}$ flex mode and C-O stretching vibration. The absorption peak at $947 \mathrm{~cm}^{-1}$ was attributed to the stretching vibration of $C=C$ or the bending vibration of some hydrogen-containing groups. This confirms the presence of the characteristic functional groups of thyme essential oil in the samples. These displaced peaks indicate the intermolecular interaction between the polymers and the thyme essential oil. Intermolecular interaction plays an important role in the encapsulating polymer and the stabilizer because it is understood as evidencing compatibility in nanocapsules.

\subsection{Thermal Analysis of the Nanocapsules}

DSC analyzed the thermal behavior of the polymeric nanocapsules to confirm the formation of the thyme essential oil nanocapsules. Figure 5a-d shows the DSC thermograms of the stabilizing and encapsulating polymers used in this process. Figure 5 a shows that the melting point of the PCL was $61^{\circ} \mathrm{C}$, similar to that reported by Wang et al. (2018) [9]. Ethylcellulose is a highly amorphous or even completely amorphous material. The $\mathrm{Tg}$ of ethylcellulose is in the range of $130-150^{\circ} \mathrm{C}$ (Figure $5 \mathrm{~b}$ ), depending on the type and measurement method used [10]. In the case of the stabilizing polymers, the thermogram of Pluronic ${ }^{\circledR}$ F-127 (Figure 5c) reveals an acute endothermic peak in the $50-70{ }^{\circ} \mathrm{C}$ range due to dehydration of this polymer. This surfactant does not show any endothermic peak or phase transition since it is an amorphous polymer and, therefore, exhibits a wide endotherm due to dehydration [12]. The PVA thermal curve (Figure 5d) presented two melting points at $55^{\circ} \mathrm{C}$ and $144{ }^{\circ} \mathrm{C}$, respectively. Studies carried out by Rebia et al. (2018) [11] indicate a similar behavior to that obtained in the present work.

Figure $5 \mathrm{e}-\mathrm{h}$ shows the thermal curves obtained for the different nanocapsules obtained by the emulsification-diffusion method. They denote that the melting points are related directly to the encapsulating polymers, regardless of the stabilizing polymer. The decrease in peak amplitude and melting temperature values can be explained as a strong interaction between PCL, ETC that is independent of the essential oil, as described by Adeli (2016) [12] in trials with Pluronic ${ }^{\circledR}$ and irbesartan. The quantitative characterization of the thermal parameters of the nanocapsules by DSC was also carried out. The ANOVA performed revealed no statistically significant difference for the $\mathrm{T}_{\mathrm{g}}$ and $\mathrm{T}_{\text {Fusion }}$ of the samples $(p>0.05)$ with the same encapsulating polymer. However, in the case of $\Delta \mathrm{H}$, there was a significant difference $(p \leq 0.05)$ due only to the stabilizing polymer. PVA values are in the range of $2.5-3 \mathrm{~kJ} / \mathrm{kg}$ and $75-81 \mathrm{~kJ} / \mathrm{kg}$ for Pluronic ${ }^{\circledR}$. The decrease in fusion enthalpy indicates a reduction in the crystallinity of the samples [11]. 

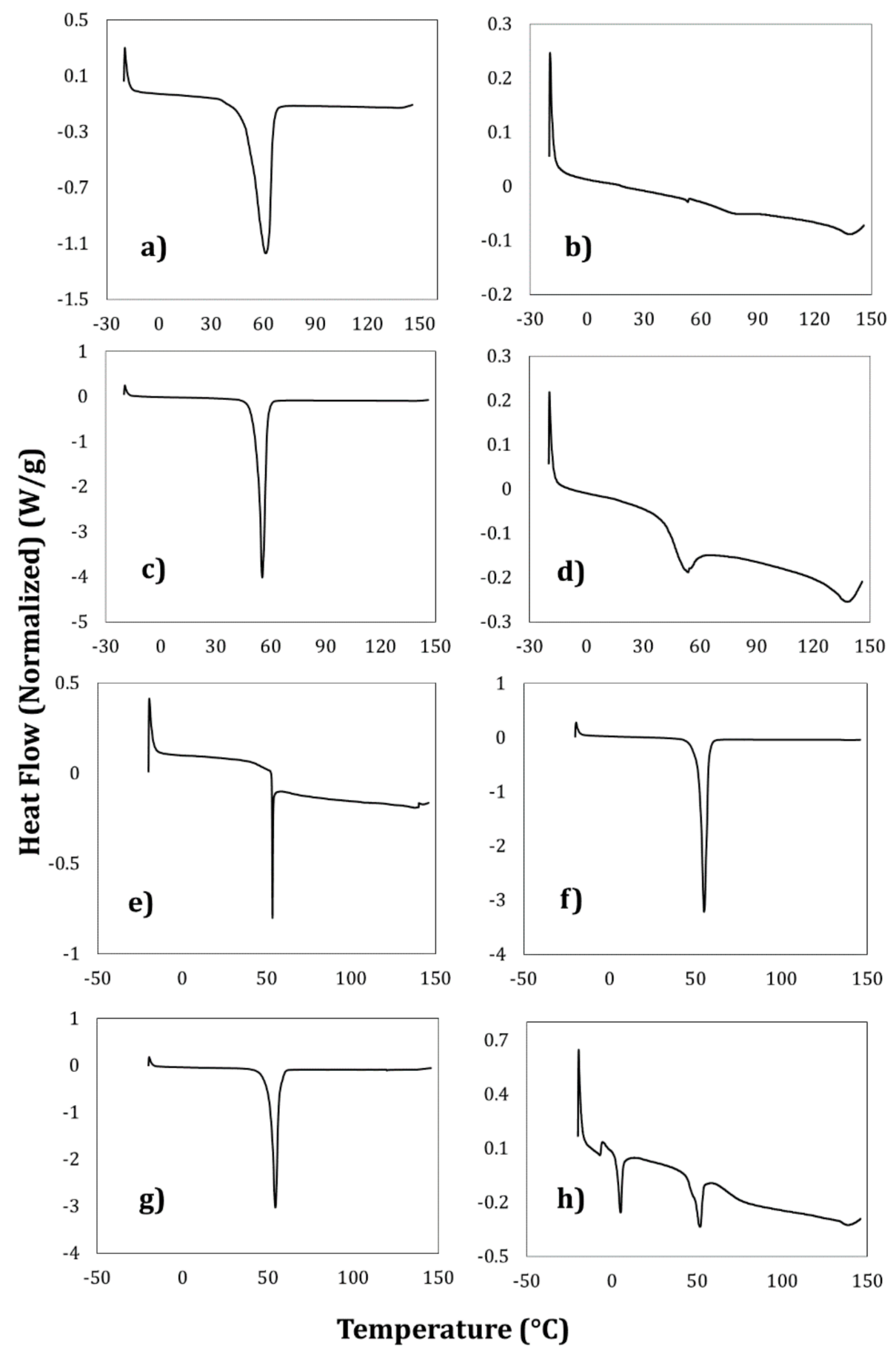

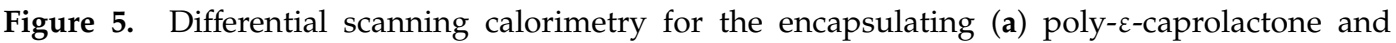
(b) ethyl cellulose polymers, and the stabilizing polymers (c) Pluronic ${ }^{\circledR}$ F-127 and (d) PVA used to form the polymeric nanocapsules: (e) ETC-PVA; (f) ETC-Pluronic ${ }^{\circledR}$ F-127, (g) PCL-Pluronic ${ }^{\circledR}$ F-127 and (h) PCL-PVA.

\subsection{Stability of the Nanocapsules}

Figure 6 presents the diffuse reflectance profiles obtained for the nanocapsules prepared by the emulsification-diffusion method. No statistically significant differences $(p>0.05)$ were detected between the samples in the different formation conditions (encapsulating and stabilizing polymers).

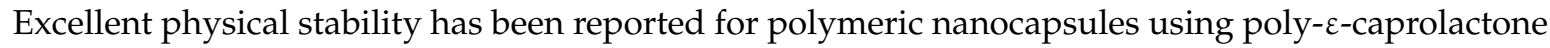
as encapsulating polymer, $\beta$-carotene as active substance (dissolved in sunflower oil), and Pluronic ${ }^{\circledR}$ 
F-127 as stabilizing polymer during storage at 4 and $25^{\circ} \mathrm{C}$ for 28 days [4]. This is consistent with the present experimental study. It is worth mentioning that in the present study, the good physical stability revealed in the diffuse reflectance studies is attributed to the difference in surface charges when Pluronic ${ }^{\circledR}$ F-127 is used and to steric effects when polyvinyl alcohol is used.

\section{Transsmision}
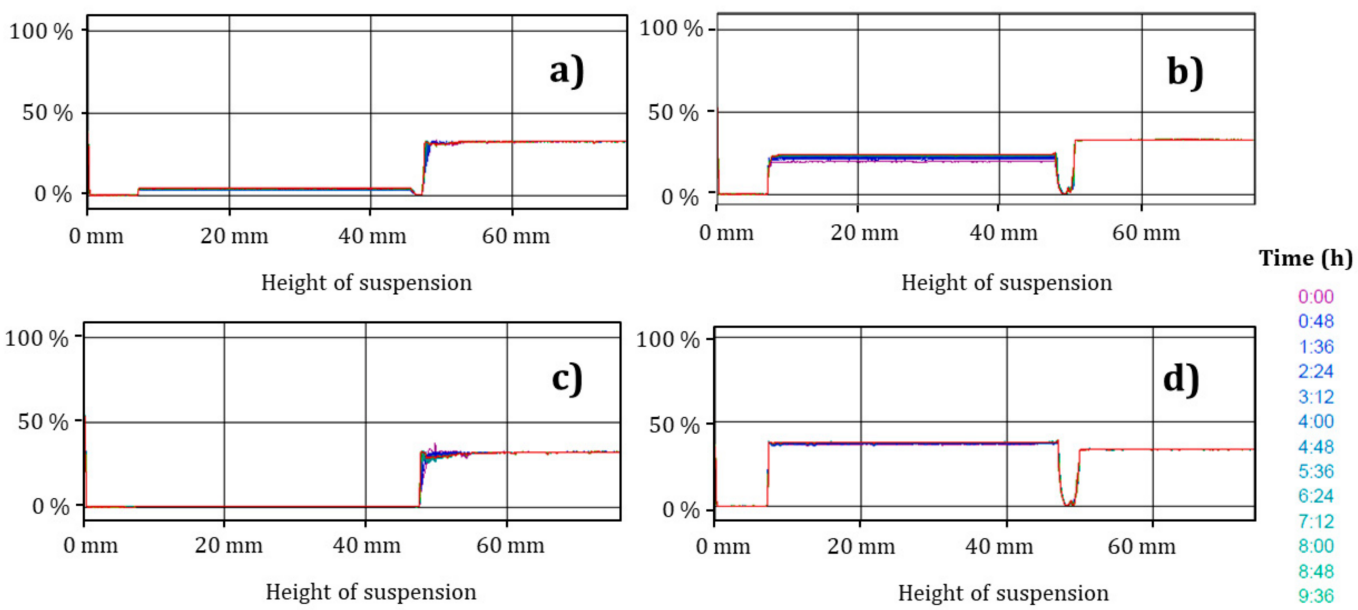

Back Scattering
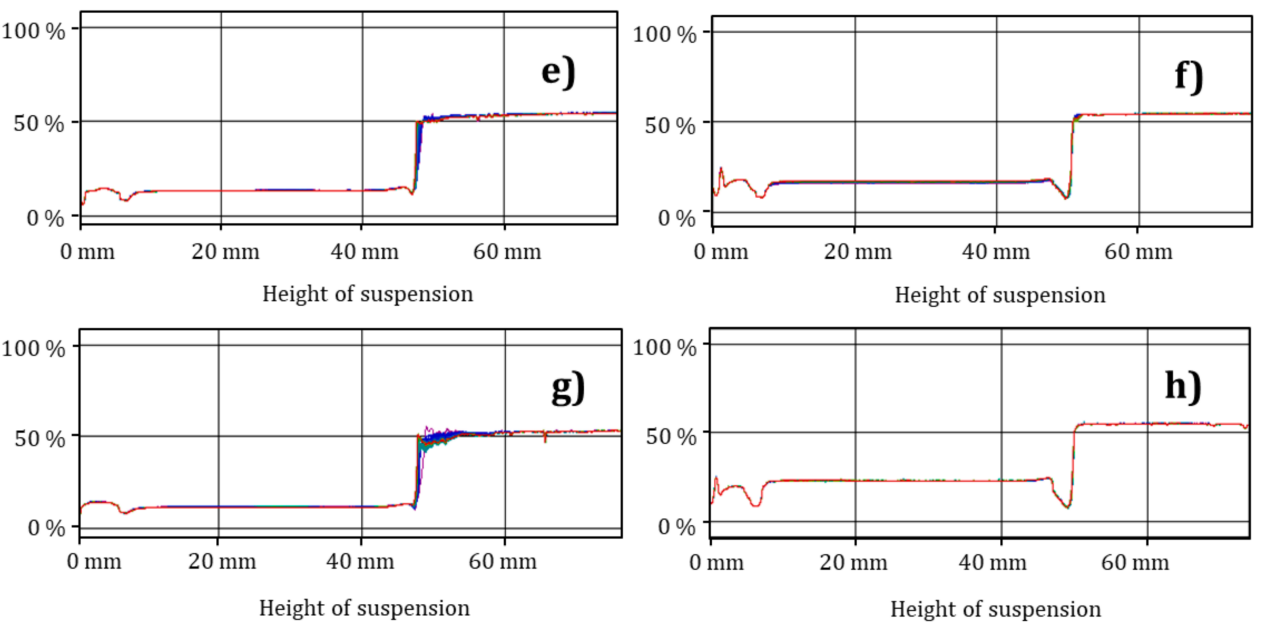

Figure 6. Diffuse reflectance: (a) and (e) ETC-PVA; (b) and (f) ETC-Pluronic ${ }^{\circledR}$ F-127; (c) and (g) PCL-Pluronic ${ }^{\circledR}$ F-127; and (d) and (h) PCL-PVA.

\subsection{Antioxidant Capacity of the Nanocapsules}

The antioxidant capacity of the polymeric nanocapsules was established using different substances since each one provides important information on the possible action mechanisms of the contained substances (Table 3). This analysis revealed that, regardless of method, no significant difference $(p>0.05)$ was found between the antioxidant capacity obtained from the polymeric nanocapsules and that of the free essential oil. This implies that, in the sizes obtained (200-500 nm), there was no increase or decrease in this property and that the antioxidant capacity evaluated depends exclusively on the active molecules contained in the essential oil [1], regardless of the polymers used (whether encapsulating or stabilizing). The gas chromatography analysis performed by Oliveira et al. (2020) [29] indicated that the main active substances present in the essential oil of thyme were a total of 28 compounds, presenting five among the most abundant: thymol (50.5\%), p-cymene $(19.4 \%), \gamma$-terpinene $(9.1 \%)$, carvacrol (5.35\%) and $\beta$-linalool (3.36\%). 
Table 3. Antioxidant capacity of the different polymeric nanocapsules obtained by the radicals ABTS, FRAP, and DPPH.

\begin{tabular}{cccc}
\hline \multirow{2}{*}{ Sample } & ABTS & FRAP & DPPH \\
\cline { 2 - 4 } & \multicolumn{2}{c}{$\boldsymbol{\mu \text { mol } _ { \text { ascorbic acid equivalents/g } } \text { (gnanostructured oil }}$} \\
\hline Thyme oil & $2010.74 \pm 43.94$ & $946.04 \pm 12.87$ & $274.74 \pm 37.71$ \\
ETC-PVA & $1924.03 \pm 74.52$ & $966.38 \pm 22.18$ & $272.79 \pm 41.46$ \\
ETC-Pluronic $^{\circledR}$ & $2135.58 \pm 33.72$ & $1071.75 \pm 19.59$ & $285.13 \pm 14.28$ \\
PCL-PVA & $1973.69 \pm 59.01$ & $1108.92 \pm 56.37$ & $280.46 \pm 20.02$ \\
PCL-Pluronic $^{\circledR}$ & $2034.40 \pm 55.28$ & $1133.06 \pm 18.97$ & $269.30 \pm 12.07$ \\
\hline
\end{tabular}

As reported in the literature, the antioxidant capacity may differ depending on the assay used. The antioxidant capacity evaluated by ABTS was greater in all cases than the results obtained by DPPH (less than $90 \%$ ) and lower than $50 \%$ on the FRAP tests $(p \leq 0.05)$. This can be correlated to the ABTS assays that determine the hydrophilic and lipophilic antioxidant activity in food and provide a highly-adequate context for the overall antioxidant content [30]. However, the DPPH and FRAP test determinations in the present study allow us to understand various chemical phenomena involved in releasing the active substances from thyme essential oil, such as chelating capacity and polymerization properties.

Several studies have analyzed the antioxidant capacity of the major compounds in thyme essential oil. Milos and Dakota (2012) [31] carried out an investigation on antioxidant synergisms and antagonisms between thymol, thymoquinone, carvacrol, and p-cymene in a model system, finding that there is an antioxidant synergism between thymol and carvacrol and antagonism between thymoquinone and p-cymene. This allows inferring the variation between the different values evaluated for antioxidant capacity depending on the method (mechanism of action).

\section{Conclusions}

It was possible to obtain physically stable polymeric nanoparticles of nanometric size (less than $500 \mathrm{~nm}$ ) with a low probability of aggregation in all cases. Thermal and I.R. analyses revealed good integration of the components in the nanostructured system, independent of the encapsulating and stabilizing polymers used. The study determined that the most adequate kinetic modeling of nanocapsule release for describing the phenomena involved is the one proposed by Korsmeyer-Peppas, which defines the release as Fickian in the nanostructured system. The nanocapsules' antioxidant capacity reveals the different action mechanisms suggested for each test, such as polymerization and chelation. It was possible to form a nanostructured system with biodegradable wall polymers (PCL and PVA) that positively impacted the physical, antioxidant, and stability properties. The potential application of the nanostructured systems obtained in this work in food processing and preservation can be inferred.

Author Contributions: R.M.G.-R.: Investigation, methodology, formal analysis, writing-original draft. H.H.-S.: Supervision, writing-review and editing. M.L.Z.-Z.: conceptualization, writing-review and editing, project administration, funding acquisition, moreover, final approval document. G.F.G.-L.: investigation, methodology. A.D.R.: Microscopy analysis and D.Q.-G.: Proposal methodology and supervision of release kinetic. B.V.-B.: Analysis of Infrared spectra data. All authors have read and agreed to the published version of the manuscript.

Funding: This research received no external funding.

Acknowledgments: M.C. Ricardo Moises Gonzalez Reza is grateful for the doctoral scholarship support from CONACYT-México (702874). The authors thank María de los Angeles Cornejo Villegas for her help during the experimental work. The authors also acknowledge the financial support for PAPIIT projects IN222520 of DAGAPA-UNAM and Internal Project-FESC PIAPI2060.

Conflicts of Interest: The authors declare that they have no known competing financial interests or personal relationships that could have appeared to influence the work reported in this paper. 


\section{References}

1. Asprea, M.; Leto, I.; Bergonzi, M.C.; Bilia, A.R. Thyme essential oil loaded in nanocochleates: Encapsulation efficiency, in vitro release study and antioxidant activity. LWT Food Sci. Technol. 2017, 77, 497-502. [CrossRef]

2. Hussein, M.; Roby, H.; Atef, M.; Selim, K.A.; Ibrahim, K. Evaluation of antioxidant activity, total phenols and phenolic compounds in thyme (Thymus vulgaris L.), sage (Salvia officinalis L.), and marjoram (Origanum majorana L.) extracts. Ind. Crop. Prod. 2013, 43, 827-831. [CrossRef]

3. Pokorný, J.; Yanishlieva, N.; Gordon, M. Antioxidants in Food: Practical Applications; Woodhead Publishing Ltd. and CRC Press LLC: Cambridge, UK, 2001.

4. González-Reza, R.M.; Quintanar-Guerrero, D.; Del Real-López, A.; Piñon-Segundo, E.; Zambrano-Zaragoza, M.L. Effect of sucrose concentration and $\mathrm{pH}$ onto the physical stability of $\beta$-carotene nanocapsules. LWT Food Sci. Technol. 2018, 90, 354-361. [CrossRef]

5. González-Reza, R.M.; Zambrano-Zaragoza, M.L.; Hernández-Sánchez, H. Polymeric Nanoparticles in Foods. In Plant Nanobionics. Nanotechnology in the Life Sciences; Springer: Berlin/Heidelberg, Germany, 2019; pp. 217-233.

6. Mora-Huertas, C.E.; Fessi, H.; Elaissari, A. Polymer-based nanocapsules for drug delivery. Int. J. Pharm. 2010, 385, 113-142. [CrossRef] [PubMed]

7. Zambrano-Zaragoza, M.L.; Mercado-Silva, E.; Gutiérrez-Cortez, E.; Castaño-Tostado, E.; Quintanar-Guerrero, D. Optimization of nanocapsules preparation by the emulsion-diffusion method for food applications. LWT Food Sci. Technol. 2011, 44, 1362-1368. [CrossRef]

8. Quintanar-Guerrero, D.; Allémann, E.; Doelker, E.; Fessi, H. Preparation and characterization of nanocapsnles from preformed polymers by a new process based on emulsification-diffusion technique. Pharm. Res. 1998, 15, 1056-1062. [CrossRef] [PubMed]

9. Wang, J.; Tian, L.; Luo, B.; Ramakrishna, S.; Kai, D.; Loh, X.J.; Yang, I.H.; Deen, G.R.; Mo, X. Engineering PCL/lignin nanofibers as an antioxidant scaffold for the growth of neuron and Schwann cell. Colloids Surf. B Biointerfaces 2018, 169, 356-365. [CrossRef] [PubMed]

10. Paulo, F.; Santos, L. Inclusion of hydroxytyrosol in ethyl cellulose microparticles: In vitro release studies under digestion conditions. Food Hydrocoll. 2018, 84, 104-116. [CrossRef]

11. Rebia, R.A.; Rozet, S.; Tamada, Y.; Tanaka, T. Biodegradable PHBH/PVA blend nanofibers: Fabrication, characterization, in vitro degradation, and in vitro biocompatibility. Polym. Degrad. Stab. 2018, 154, 124-136. [CrossRef]

12. Adeli, E. The use of supercritical anti-solvent (SAS) technique for preparation of Irbesartan-Pluronic ${ }^{\circledR} \mathrm{F}-127$ nanoparticles to improve the drug dissolution. Powder Technol. 2016, 298, 65-72. [CrossRef]

13. Siepmann, J.; Peppas, N.A. Modeling of drug release from delivery systems based on hydroxypropyl methylcellulose (HPMC). Adv. Drug Deliv. Rev. 2012, 64, 163-174. [CrossRef]

14. Zambrano-Zaragoza, M.L.; Quintanar-Guerrero, D.; Del Real, A.; Piñon-Segundo, E.; Zambrano-Zaragoza, J.F. The release kinetics of $\beta$-carotene nanocapsules/xanthan gum coating and quality changes in fresh-cut melon (cantaloupe). Carbohydr. Polym. 2017, 157, 1874-1882. [CrossRef] [PubMed]

15. Zambrano-Zaragoza, M.L.; Mercado-Silva, E.; Del Real, L.A.; Gutiérrez-Cortez, E.; Cornejo-Villegas, M.A.; Quintanar-Guerrero, D. The effect of nano-coatings with $\alpha$-tocopherol and xanthan gum on shelf-life and browning index of fresh-cut "red Delicious" apples. Innov. Food Sci. Emerg. Technol. 2014, 22, 188-196. [CrossRef]

16. González-Reza, R.M.; Quintanar-Guerrero, D.; Flores-Minutti,J.J.; Gutiérrez-Cortez, E.; Zambrano-Zaragoza, M.L. Nanocapsules of $\beta$-carotene: Thermal degradation kinetics in a scraped surface heat exchanger (SSHE). LWT Food Sci. Technol. 2015, 60, 124-130. [CrossRef]

17. Soares, P.I.P.; Sousa, A.I.; Silva, J.C.; Ferreira, I.M.M.; Novo, C.M.M.; Borges, J.P. Chitosan-based nanoparticles as drug delivery systems for doxorubicin: Optimization and modelling. Carbohydr. Polym. 2016, 147, $304-312$. [CrossRef] [PubMed]

18. Re, R.; Pellegrini, N.; Proteggente, A.; Pannala, A.; Yang, M.; Rice-Evans, C. Antioxidant Activity Applying An Improved Abts Radical. Free Radic. Biol. Med. 1999, 26, 1231-1237. [CrossRef]

19. Brand-Williams, W.; Cuvelier, M.E.; Berset, C. Use of a free radical method to evaluate antioxidant activity. LWT Food Sci. Technol. 1995, 28, 25-30. [CrossRef] 
20. Benzie, I.F.F.; Strain, J.J. The ferric reducing ability of plasma (FRAP) as a measure of "antioxidant power": The FRAP assay. Anal. Biochem. 1996, 239, 70-76. [CrossRef]

21. Galindo-Pérez, M.J.; Quintanar-Guerrero, D.; de los Ángeles Cornejo-Villegas, M.; de la Luz Zambrano-Zaragoza, M. Optimization of the emulsification-diffusion method using ultrasound to prepare nanocapsules of different food-core oils. LWT Food Sci. Technol. 2018, 87, 333-341. [CrossRef]

22. Gupta, S.S.; Ghosh, M. Preparation and characterisation of protein based nanocapsules of bioactive lipids. J. Food Eng. 2014, 121, 64-72. [CrossRef]

23. Cano-Sarmiento, C.; Téllez-Medina, D.I.; Viveros-Contreras, R.; Cornejo-Mazón, M.; Figueroa-Hernández, C.Y.; García-Armenta, E.; Alamilla-Beltrán, L.; García, H.S.; Gutiérrez-López, G.F. Zeta Potential of Food Matrices. Food Eng. Rev. 2018, 10, 113-138. [CrossRef]

24. Elbasuney, S. Sustainable steric stabilization of colloidal titania nanoparticles. Appl. Surf. Sci. 2017, 409, $438-447$. [CrossRef]

25. Noronha, C.M.; Granada, A.F.; de Carvalho, S.M.; Lino, R.C.; Matheus, M.V.; Barreto, P.L.M. Optimization of $\alpha$-tocopherol loaded nanocapsules by the nanoprecipitation method. Ind. Crops Prod. 2013, 50, 896-903. [CrossRef]

26. Sotelo-Boyás, M.E.; Correa-Pacheco, Z.N.; Bautista-Baños, S.; Corona-Rangel, M.L. Physicochemical characterization of chitosan nanoparticles and nanocapsules incorporated with lime essential oil and their antibacterial activity against food-borne pathogens. LWT Food Sci. Technol. 2017, 77, 15-20. [CrossRef]

27. Lin, L.; Zhu, Y.; Cui, H. LWT-Food Science and Technology Electrospun thyme essential oil/gelatin nano fi bers for active packaging against Campylobacter jejuni in chicken. LWT Food Sci. Technol. 2018, 97, 711-718. [CrossRef]

28. Elzein, T.; Nasser-Eddine, M.; Delaite, C.; Bistac, S.; Dumas, P. FTIR study of polycaprolactone chain organization at interfaces. J. Colloid Interface Sci. 2004, 273, 381-387. [CrossRef]

29. Oliveira, R.C.; Carvajal-Moreno, M.; Correa, B.; Rojo-Callejas, F. Cellular, physiological and molecular approaches to investigate the antifungal and anti-aflatoxigenic effects of thyme essential oil on Aspergillus flavus. Food Chem. 2020, 315, 126096. [CrossRef]

30. Cano, A.; Arnao, M.B. ABTS/TEAC (2, 2' -azino-bis (3-ethylbenzothiazoline-6-sulfonic acid)/Trolox ${ }^{\circledR}$-Equivalent Antioxidant Capacity) radical scavenging mixed-mode assay. In Measurement of Antioxidant Activity $\mathcal{E}$ Capacity: Recent Trends and Applications; John Wiley \& Sons Ltd.: Pondicherry, India, 2018; pp. 117-139.

31. Milos, M.; Makota, D. Investigation of antioxidant synergisms and antagonisms among thymol, carvacrol, thymoquinone and p-cymene in a model system using the Briggs-Rauscher oscillating reaction. Food Chem. 2012, 131, 296-299. [CrossRef]

Publisher's Note: MDPI stays neutral with regard to jurisdictional claims in published maps and institutional affiliations.

(C) 2020 by the authors. Licensee MDPI, Basel, Switzerland. This article is an open access article distributed under the terms and conditions of the Creative Commons Attribution (CC BY) license (http://creativecommons.org/licenses/by/4.0/). 